\title{
Reflets
}

Revue d'intervention sociale et communautaire

\section{5 ans plus tard... et toujours nécessaires! Les approches structurelles dans le champ de l'intervention sociale}

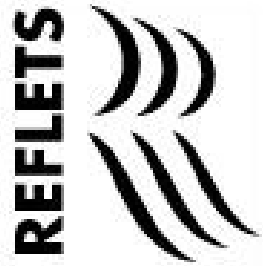

\section{Simon Lapierre et Joscelyne Levesque}

Volume 19, numéro 1, printemps 2013

Approches structurelles et intervention sociale

URI : https://id.erudit.org/iderudit/1018041ar

DOI : https://doi.org/10.7202/1018041ar

Aller au sommaire du numéro

Éditeur(s)

Reflets, Revue d'intervention sociale et communautaire

ISSN

1203-4576 (imprimé)

1712-8498 (numérique)

Découvrir la revue

Citer cet article

Lapierre, S. \& Levesque, J. (2013). 25 ans plus tard... et toujours nécessaires! Les approches structurelles dans le champ de l'intervention sociale. Reflets, 19(1), 38-64. https://doi.org/10.7202/1018041ar

\section{Résumé de l'article}

De manière générale, les approches structurelles reposent sur les deux principes suivants : 1 . reconnaître que les situations individuelles sont intimement reliées aux structures politiques, sociales et économiques; 2. reconnaître que la réalisation de changements significatifs dans les situations auxquelles les individus sont confrontés exige aussi des changements dans les structures politiques, sociales et économiques et que de tels changements sont possibles. Ainsi, les approches structurelles sont considérées comme étant des approches critiques, conflictuelles et radicales, tout comme les approches antiracistes et antioppressives, ainsi que certaines approches féministes. Elles s'appuient sur des valeurs de justice sociale, d'égalité et de solidarité. Le présent article s'intéresse aux dimensions théoriques et pratiques, mais se penche d'abord sur les défis auxquels sont confrontées les intervenantes qui souscrivent aux approches structurelles dans l'intervention sociale contemporaine. La deuxième partie de l'article présente les principaux éléments d'analyse sur lesquels s'appuient les approches structurelles, ainsi que leur pertinence dans le contexte actuel. La troisième partie de l'article se penche davantage sur l'application de ces approches en fonction de trois cibles d'intervention (individuelle, groupe et collective) ainsi que sur les habiletés d'intervention nécessaires.
Tous droits réservés @ Reflets, Revue d’intervention sociale et communautaire, 2013
Ce document est protégé par la loi sur le droit d'auteur. L'utilisation des services d'Érudit (y compris la reproduction) est assujettie à sa politique d'utilisation que vous pouvez consulter en ligne. 


\section{5 ans plus tard... et toujours nécessaires!}

\section{Les approches structurelles dans le champ de l'intervention sociale}

Simon Lapierre, Ph. D.

Professeur agrégé, École de service social, Université d'Ottawa

Joscelyne Levesque, MSS

Coordonnatrice de la formation pratique et des stages

École de service social, Université d'Ottawa

\section{Résumé}

De manière générale, les approches structurelles reposent sur les deux principes suivants : 1. reconnaitre que les situations individuelles sont intimement reliées aux structures politiques, sociales et économiques; 2 . reconnaitre que la réalisation de changements significatifs dans les situations auxquelles les individus sont confrontés exige aussi des changements dans les structures politiques, sociales et économiques et que de tels changements sont possibles. Ainsi, les approches structurelles sont considérées comme étant des approches critiques, conflictuelles et radicales, tout comme les approches antiracistes et antioppressives, ainsi que certaines approches féministes. Elles s'appuient sur des valeurs de justice sociale, d'égalité et de solidarité. Le présent article s'intéresse aux dimensions théoriques et pratiques, mais se penche d'abord sur les défis auxquels sont confrontées les intervenantes ${ }^{1}$ qui souscrivent aux approches structurelles dans l'intervention sociale contemporaine. La deuxième partie de 
l'article présente les principaux éléments d'analyse sur lesquels s'appuient les approches structurelles, ainsi que leur pertinence dans le contexte actuel. La troisième partie de l'article se penche davantage sur l'application de ces approches en fonction de trois cibles d'intervention (individuelle, groupe et collective) ainsi que sur les habiletés d'intervention nécessaires.

Mots clés : Approches structurelles, changement social, droits, intervention individuelle, intervention de groupe, intervention collective, justice sociale, oppression, système de domination

\section{Abstract}

Structural social work practices are based on the following two principles: 1. the recognition that individual situations are linked to political, social and economic structures, and 2. the recognition that significant changes in these individual situations also require changes at the political, social and economic levels - and the recognition that such changes are possible. Therefore, structural social work practices are critical and radical approaches, rooted in conflict theories - as are anti-racist and anti-oppressive practices, as well as some feminist practices. They are rooted in values such as social justice, equality, and solidarity. This paper addresses both theoretical and practical issues, but first considers contemporary challenges for workers subscribing to a structural perspective. The second section focuses on the main elements that need to be taken into account in a structural analysis, while the last section looks at structural social work practice with individuals, groups and communities.

Key words : Structural social work, social change, rights, social work intervention, groupwork, community work, social justice, oppression, domination.

\section{Introduction}

Elle [l'approche structurelle] n'est pas un dogme, mais une hypothèse de travail qui, pour demeurer 
"Considérant les développements des deux dernières décennies, nous estimons qu'il est maintenant plus approprié de faire référence aux approches structurelles plutôt que de prétendre qu'il existe une approche unique, tout particulièrement dans le contexte actuel." pertinente et valable dans l'intervention sociale, doit être continuellement confrontée aux tests de la pratique. Elle requiert une certaine énergie et comporte un certain risque (Moreau, 1987, p. 243)

"L'approche structurelle en travail social : implications pratiques d'une approche intégrée conflictuelle » (Moreau, 1987) est un texte marquant et incontournable pour toutes celles qui s'intéressent aux approches structurelles et à leur application dans le champ de l'intervention sociale. Dans l'exergue ci-dessus, Moreau souligne que pour demeurer pertinente et valable dans l'intervention, l'approche structurelle devrait être continuellement confrontée aux tests de la pratique. Vingt-cinq ans après la publication de cet article, le paysage de l'intervention a changé de manière significative, tout comme le contexte politique, social et économique dans lequel les interventions s'inscrivent. Le présent article revisite donc les approches structurelles. Considérant les développements des deux dernières décennies, nous estimons qu'il est maintenant plus approprié de faire référence aux approches structurelles plutôt que de prétendre qu'il existe une approche unique, tout particulièrement dans le contexte actuel.

De manière générale, les approches structurelles reposent sur les deux principes suivants : 1 . reconnaitre que les situations individuelles sont intimement reliées aux structures politiques, sociales et économiques; 2 . reconnaitre que la réalisation de changements significatifs dans les situations auxquelles les individus sont confrontés exige aussi des changements dans les structures politiques, sociales et économiques et reconnaitre que de tels changements sont possibles (Murray et Hick, 2010). Ainsi, les approches structurelles sont considérées comme étant des approches critiques, conflictuelles et radicales, tout comme les approches antiracistes et antioppressives, ainsi que certaines approches féministes. Elles s'appuient sur des valeurs de justice sociale, d'égalité et de solidarité (Auclair, 2000; Thibault, 2011).

Si certaines personnes œuvrant dans le champ de l'intervention sociale adhèrent aux deux principes mentionnés ci-dessus et les 
placent au centre de leur pratique, il n'en demeure pas moins que les approches structurelles se sont toujours situées à la marge, essentiellement au sein du mouvement communautaire, puisque le travail social a plutôt été dominé par des approches centrées sur les individus et les familles, plus conservatrices et prônant le contrôle et l'ordre social (Favreau, 2000; Mullaly, 2007; Mendes, 2009; Lundy, 2011). Ainsi, dans notre travail de formation avec des étudiantes en travail social, nous constatons que plusieurs d'entre elles ne perçoivent pas les liens entre les dimensions individuelles et structurelles des problèmes; et celles qui y parviennent se sentent souvent impuissantes face à leur application. Dans le même sens, Murray et Hick (2010) soulignent que les écrits sur les approches structurelles ont souvent été critiqués pour avoir mis l'accent sur les théories et pour avoir porté une attention limitée aux dimensions plus pratiques de l'intervention sociale dans une perspective structurelle.

Divisé en trois parties, le présent article porte donc sur les dimensions tant pratiques que théoriques des approches structurelles dans l'intervention sociale contemporaine. La première partie expose les défis auxquels sont confrontées les intervenantes qui y souscrivent. Les principaux éléments d'analyse sur lesquels s'appuient les approches structurelles, ainsi que leur pertinence dans le contexte actuel, font l'objet de la deuxième partie. La troisième partie se penche davantage sur l'application de ces approches en fonction de trois cibles d'intervention (l'individu, le groupe et la collectivité) ainsi que sur les habiletés d'intervention nécessaires.

\section{Des défis dans l'intervention sociale contemporaine}

$\mathrm{Si}$, comme nous venons de le mentionner, les approches structurelles ont toujours été situées à la marge dans le champ de l'intervention sociale, les tenants de ces approches sont confrontés à des défis particuliers dans le contexte politique, social et économique actuel.En 1987, dans la conclusion de son article déjà mentionné, Moreau faisait référence au « vent de conservatisme » 
"...Moreau faisait référence au "vent de conservatisme » qui commençait à souffler sur les pays occidentaux industrialisés. Vingt-cinq ans plus tard, il semble que ce "vent " soit omniprésent. Et il souffle fort! " qui commençait à souffler sur les pays occidentaux industrialisés. Vingt-cinq ans plus tard, il semble que ce " vent » soit omniprésent. Et il souffle fort!

Dans un contexte politique et social plus progressiste, les politiques mises en place favorisent l'équité et la justice sociale, en préconisant notamment l'universalité des services et une meilleure redistribution des ressources. Cependant, depuis le début des années 1980, le discours néolibéraliste a mis de l'avant l'idée que toute activité devait être soumise aux lois du marché et que l'intervention de l'état devait être réduite à son minimum. Les choix découlant de cette idéologie favorisent les choix individuels et l'enrichissement personnel, au détriment du bien commun (Vaillancourt, 2001; Gill, 2004). Ainsi, même la notion de droits, qui a été mobilisée par les mouvements populaires, syndicaux, étudiants et autres, est souvent réduite à une dimension individuelle dans le contexte néolibéral actuel, faisant ainsi primer les droits individuels sur les droits collectifs (Shragge, 2006).

En plus de ces réalités, les intervenantes qui souscrivent aux approches structurelles sont confrontées à trois tendances lourdes dans le champ de l'intervention sociale : 1. l'individualisation et la psychologisation; 2. la professionnalisation et la spécialisation; 3. les pratiques fondées sur les données probantes et les systèmes de surveillance et de régulation.

\section{Individualisation et psychologisation des problèmes sociaux et de l'intervention sociale}

Dans le contexte actuel, les difficultés auxquelles les individus et les groupes sont confrontés, entre autres, la pauvreté économique, l'itinérance et la violence, sont souvent réduites à leur dimension individuelle, voire psychologique. Ainsi, ces difficultés résulteraient essentiellement de "déficits" chez les individus concernés, occultant ainsi la contribution de facteurs politiques, sociaux et économiques. Et les pistes envisagées pour changer la situation misent sur le « traitement » et sur la responsabilisation individuelle.

Il semble que cette tendance à l'individualisation des problèmes sociaux et de l'intervention sociale soit même présente au sein de 
"Dans le contexte actuel, les difficultés auxquelles les individus et les groupes sont confrontés, entre autres, la pauvreté économique, l'itinérance et la violence, sont souvent réduites à leur dimension individuelle, voire psychologique. " plusieurs organismes communautaires (René, 2009), et ce, en dépit du fait que le mouvement communautaire ait été très critique face aux approches centrées sur les individus et les familles, plus souvent associées aux pratiques institutionnelles. René (2009) note une accentuation de l'individualisation dans l'intervention au sein de nombreux organismes communautaires québécois, se traduisant par des interventions qui ont «trop souvent des visées d'effets individualisés sur les personnes rejointes"sans nécessairement établir de "passerelles entre des objectifs visant les besoins des individus et des objectifs d'intervention qui peuvent avoir un impact plus direct sur la communauté et sa vie démocratique " (p. 116). L'auteur soutient que cette tendance serait présente, notamment, au sein d'organismes communautaires qui travaillent avec des familles et des jeunes, malgré leur adhésion à un discours prônant une approche globale, une prise en charge collective et une participation démocratique. René conclut ainsi à une certaine inadéquation entre les cadres de référence et les pratiques réelles :

Les formes collectives d'action et d'appropriation occupent une place effective assez ténue dans le quotidien de bon nombre d'organismes jeunesse et famille... Même si les approches empruntées à l'éducation populaire sont présentes dans le discours des regroupements d'organismes, sur le terrain, un fossé sépare trop souvent les cadres de référence des pratiques réelles. (2009, p. 115)

Cette inadéquation entre les cadres de référence et les pratiques réelles a aussi été notée par Prud'homme (2011) dans un article portant sur l'intervention en maisons d'hébergement pour femmes victimes de violence conjugale (voir aussi Krane et Carlton, 2012, sur l'intervention avec les femmes victimes de violence conjugale et leurs enfants). Prud'homme met en garde contre certains glissements vers des interventions psychologisantes, qui vont à l'encontre de l'analyse féministe de la violence et des principes à la base de l'intervention féministe. Cela s'observe notamment lorsque les réactions de victimisation des femmes sont confondues avec la dépendance affective ou avec toute autre 
affectation psychologique, plutôt que d'être perçues comme des conséquences de la violence.

Les approches structurelles accordent une place centrale aux expériences individuelles et n'évacuent pas complètement la notion de responsabilité individuelle — l'évacuation complète de cette notion amènerait une représentation des individus comme des victimes passives, incapables de faire des choix (Mullender, 1996), mais compromettrait aussi toute forme de cohésion sociale au sein d'un groupe ou d'une collectivité. Cette tendance à réduire les difficultés auxquelles les individus et les groupes sont confrontés à leur dimension individuelle ou psychologique relève néanmoins d'une logique qui s'oppose aux analyses structurelles, puisqu'elle occulte les dimensions politique, sociale et économique.

\section{Professionnalisation et spécialisation de l'intervention sociale}

"Une autre tendance marquée dans le champ de l'intervention sociale est celle de la professionnalisation de l'intervention, laquelle positionne les intervenantes comme des « expertes " qui dispensent des " services" à une "clientèle " identifiée en fonction d'une "problématique " particulière. "
Une autre tendance marquée dans le champ de l'intervention sociale est celle de la professionnalisation de l'intervention, laquelle positionne les intervenantes comme des « expertes » qui dispensent des «services" à une "clientèle » identifiée en fonction d'une "problématique " particulière. Cette tendance serait présente, notamment, au sein du mouvement communautaire, même si elle s'inscrit dans une logique différente de celle qui a mené à la création de plusieurs organismes communautaires qui misaient plutôt sur les solidarités horizontales et sur l'autodétermination des individus, des groupes et des collectivités (Mullender et Ward, 1991). Par exemple, Prud'homme (2011) soutient que cette tendance est présente dans les maisons d'hébergement pour femmes victimes de violence conjugale. Elle expose d'ailleurs les liens étroits entre la professionnalisation, l'individualisation et la psychologisation :

On fait face actuellement à une "professionnalisation " de l'aide aux victimes qui place davantage les femmes dans un rapport d'experte à aidée. On risque alors de perdre de vue l'aspect social de la violence conjugale et la défense des droits, pour mettre davantage l'accent sur 
la dimension personnelle des problèmes de la femme. En fait, avec la "professionnalisation" de l'aide aux victimes, il y a une valorisation sociale de l'évaluation clinique et du traitement personnalisé. Par le fait même, on assiste à une dévalorisation de l'analyse féministe des difficultés présentées par les femmes, cette dernière étant vue, dans ce contexte, comme dépassée et trop éloignée de la détresse individuelle (p. 187).

"...cette

professionnalisation

s'accompagne générale-

ment d'une pression à

la spécialisation, laquelle

encourage les intervenantes

à développer une

" expertise " de plus en

plus pointue en fonction

d'une "clientèle » ou

d'une "problématique "

particulière, plutôt que de

miser sur leur rôle comme

généralistes. "
Cette professionnalisation de l'intervention sociale relève d'une logique qui s'oppose aux approches structurelles. En effet, cette tendance établit clairement une distinction entre la personne aidée et la personne aidante, mais impose également une distance hiérarchique qui se traduit même dans le vocabulaire privilégié. À ce propos, St-Amand (2000, p. 38) souligne que « les mots utilisés [dans le domaine de la relation d'aide] reflètent la distance entre les uns et les autres et, par conséquent, identifient la déviance des uns et la supériorité des autres".

Par ailleurs, cette professionnalisation s'accompagne généralement d'une pression à la spécialisation, laquelle encourage les intervenantes à développer une " expertise " de plus en plus pointue en fonction d'une « clientèle » ou d'une " problématique » particulière, plutôt que de miser sur leur rôle comme généralistes. Cela ne favorise pas le développement des approches structurelles, dans la mesure où celles-ci exigent une capacité à appréhender les multiples réalités auxquelles les individus et les groupes sont confrontés, dans leur globalité, et à intervenir à plusieurs niveaux (individus, groupes et collectivités).

\section{Pratiques fondées sur les données probantes et systèmes de surveillance et de régulation des pratiques}

Le discours faisant la promotion des pratiques fondées sur des données probantes, qui tire ses origines du domaine médical et qui est particulièrement influant dans le champ de la santé publique, occupe une place grandissante dans le champ de l'intervention sociale. Couturier et Carrier (2003, p. 68) soutiennent même que 
"Il semble toutefois nécessaire de remettre en question le statut hégémonique que les tenantes des pratiques fondées sur les données probantes accordent généralement aux connaissances produites dans une logique positiviste, niant ainsi la valeur d'autres formes de connaissances, incluant les savoirs issus de l'expérience des individus, des groupes et des collectivités." ce discours « se pose de plus en plus en condition du travail en travail social ». Ce discours n'est pas étranger au contexte politique, social et économique actuel, ainsi qu'aux sources et aux modes de financement des programmes. Il est aussi étroitement lié à la mise en place de systèmes sophistiqués de collecte de données au sein même des milieux d'intervention.

Les tenantes des approches structurelles reconnaissent la nécessité du développement de connaissances, notamment parce qu'elles permettent de démontrer l'existence de certaines formes d'inégalités dans la société, ainsi que leurs conséquences sur les groupes concernés. L'évaluation de l'intervention est aussi une pratique encouragée. Il semble toutefois nécessaire de remettre en question le statut hégémonique que les tenantes des pratiques fondées sur les données probantes accordent généralement aux connaissances produites dans une logique positiviste, niant ainsi la valeur d'autres formes de connaissances, incluant les savoirs issus de l'expérience des individus, des groupes et des collectivités (Couturier et Carrier, 2003; Pease, 2009). Le discours qui fait la promotion des pratiques fondées sur les données probantes contribue ainsi à l'accentuation et à la légitimation d'une distance hiérarchique entre la personne aidée et la personne aidante.

Par ailleurs, cette perspective ne favorise pas le développement des approches structurelles, compte tenu de la complexité qui sous-tend l'évaluation des impacts d'interventions individuelles ou d'actions collectives visant ultimement des changements politiques, sociaux ou économiques - il est beaucoup plus simple d'évaluer les impacts d'une intervention visant la modification d'un comportement chez un individu. De plus, même si les intervenantes qui s'inscrivent dans une perspective structurelle doivent constamment remettre en question leurs pratiques, les organismes ne disposent pas nécessairement des ressources nécessaires à la réalisation d'une évaluation dont les résultats seraient reconnus comme étant des données probantes.

Et si les systèmes d'informations peuvent effectivement contribuer au développement des connaissances et à l'amélioration des interventions, ils sont souvent utilisés comme instruments de 
surveillance et de régulation des pratiques (Parton, 2006). Cela peut limiter l'autonomie et la flexibilité des intervenantes.

\section{Analyse : des réalités individuelles aux systèmes de domination et d'oppression}

"...les approches

structurelles se

fondent sur une vision conflictuelle de la société, qui reconnait que les groupes qui la composent ont des intérêts divergents, souvent même contradictoires. "

\section{"Contrairement}

aux approches qui privilégient le contrôle, l'adaptation et

l'insertion sociale dans le but de maintenir l'ordre social, les approches structurelles ont une visée de changement social. »
Moreau (1987) précise que l'approche structurelle en est une conflictuelle. En effet, les approches structurelles se fondent sur une vision conflictuelle de la société, qui reconnait que les groupes qui la composent ont des intérêts divergents, souvent même contradictoires (Mullaly, 2007;2010; Lundy,2011). En privilégiant les intérêts de certains groupes au détriment d'autres groupes, les structures politiques, sociales et économiques maintiennent ces derniers en situation d'oppression; cette dernière est souvent à la base des difficultés rencontrées par les individus et les groupes, et elle contribue au maintien de ces difficultés. C'est dans cette perspective que les tenantes des approches structurelles reconnaissent que les situations individuelles sont intimement reliées aux structures politiques, sociales et économiques.

L'angle d'analyse des problèmes sociaux privilégiée par les tenantes des approches structurelles est donc très différent de celui que privilégient les tenantes de plusieurs autres approches populaires dans le champ de l'intervention sociale. Par exemple, les approches qui s'inscrivent dans une perspective psychodynamique, comportementale ou fonctionnaliste situent les causes des difficultés rencontrées par les individus dans les "déficiences » ou les «déviances » individuelles ou encore dans l'organisation ou le fonctionnement de la société (Payne, 2005).

De plus, pour les intervenantes sociales préoccupées de justice sociale, cette reconnaissance des intérêts divergents et des situations d'oppression dans lesquelles se retrouvent certains groupes met en évidence la nécessité du changement social. Contrairement aux approches qui privilégient le contrôle, l'adaptation et l'insertion sociale dans le but de maintenir l'ordre social, les approches structurelles ont une visée de changement social. En 
effet, la réalisation de changements significatifs dans les situations auxquelles les individus sont confrontés exige des changements radicaux dans les structures politiques, sociales et économiques.

\section{Oppression}

"...pour voir

et comprendre ce

phénomène il est

nécessaire d'avoir une

vision macroscopique,

puisque les individus

sont toujours opprimés

en fonction de leur

appartenance à certains

groupes sociaux. "
Mullaly (2007; 2010), l'un des auteurs contemporains les plus importants dans le domaine, considère que le concept d'oppression est à la base des approches structurelles : «The primary focus of structural social work is oppression. [...] Most structural social work practice is carried out with, or on behalf of, oppressed people.» (2007, p. 252)

À cet égard, Frye (1983) soutient qu'une personne est en situation d'oppression lorsque ses choix sont limités et lorsque ses mouvements sont restreints par un ensemble de forces et de barrières qui sont reliées entre elles. Ces forces et ces barrières ne sont ni occasionnelles, ni accidentelles, ni naturelles, même si elles se présentent souvent ainsi, et elles sont donc évitables. De plus, Frye explique que pour voir et comprendre ce phénomène il est nécessaire d'avoir une vision macroscopique, puisque les individus sont toujours opprimés en fonction de leur appartenance à certains groupes sociaux.

Dans le même sens, Mullaly (2010) souligne que même s’ils sont tous confrontés à certaines restrictions et peuvent vivre des frustrations ou de la souffrance, tous ces individus ne sont pas nécessairement en situation d'oppression :

What determines oppression is when a person is blocked from opportunities to self-development, is excluded from full participation in society, does not have certain rights that the dominant group takes for granted, or is assigned a secondclass citizenship, not because of individual talent, merit, or failure, but because of his or her membership in a particular group or category of people. (p. 40) 
Il existe diverses manifestations de l'oppression. En effet, elle ne se manifeste pas de la même façon pour tous les groupes ou même pour tous les individus à l'intérieur d'un même groupe. Young (1990) a identifié cinq principales manifestations de l'oppression : l'exploitation; la marginalisation; l'impuissance; l'impérialisme culturel; et la violence. À cette liste, il est possible d'ajouter l'exclusion, la discrimination et l'abnégation. Ces manifestations se traduisent à travers les attitudes et les actions d'individus, mais également à travers les discours politiques, ainsi qu'à travers les politiques et les pratiques sociales et économiques.

Cette analyse exige donc une bonne connaissance des problèmes sociaux, incluant la dimension historique et les dimensions politiques, sociales et économiques qui ont un impact sur les conditions de vie de certains groupes sociaux. C'est ainsi que les militantes et les chercheuses féministes ont démontré que les choix des femmes sont limités et que leurs mouvements sont souvent restreints par un ensemble de forces et de barrières qui ne sont ni occasionnelles, ni accidentelles, ni naturelles (Dominelli, 2002). Les violences faites aux femmes, incluant les agressions à caractère sexuel, la violence conjugale et les fémicides, constituent l'une des nombreuses manifestations de l'oppression sexiste et patriarcale (Mullender, 2002; Coderre et Hart, 2003; Johnson et Dawson, 2011). L'oppression vécue par les peuples autochtones, laquelle prend racine dans un contexte historique colonialiste, a aussi été documentée (Grammond, 2006; Freeman, 2007).

Les personnes issues de minorités ethnoculturelles ou de minorités sexuelles, les personnes en situation de pauvreté ou en situation de handicap, ainsi que les personnes âgées sont aussi confrontées à un ensemble de forces et de barrières qui limitent leurs choix et restreignent leurs mouvements. Les systèmes d'oppression en cause sont le racisme, l'hétérosexisme, le capitalisme, le capacitisme et l'âgisme. Malgré leurs choix limités et les contraintes érigées en systèmes, les individus ne sont pas des victimes passives; ils sont des acteurs sociaux qui peuvent, entre autres, choisir, décider, remettre en question et s'objecter (Thompson, 2001; Mullender, 2003). 
"...il n'est pas facile

d'appréhender les

systèmes d'oppression

et leurs différents

mécanismes. En effet,

il est essentiel de

reconnaitre que les

manifestations de

l'oppression, si elles

peuvent parfois être

violentes et spectaculaires, sont souvent beaucoup

plus subtiles et

insidieuses. ”
Par ailleurs, il n'est pas facile d'appréhender les systèmes d'oppression et leurs différents mécanismes. En effet, il est essentiel de reconnaitre que les manifestations de l'oppression, si elles peuvent parfois être violentes et spectaculaires, sont souvent beaucoup plus subtiles et insidieuses (Frye, 1983). Par exemple, Bastien Charlebois (2011) identifie en s'inspirant des travaux de Fish (2006) un certain nombre de processus et de gestes par lesquels opère l'hétérosexisme. Ils incluent la présomption d'hétérosexualité, l'injonction au silence, l'appel à l'assimilation et le langage infériorisant. Bastien Charlebois soutient que

[c]e que l'hétérosexisme relève, c'est que les injustices commises à l'endroit de personnes LGBT ne se résument pas à quelques rares débordements émotifs et gestes d'agression. Elles s'étendent à tous les petits gestes et allusions du quotidien qui dévalorisent leur vie amoureuse et sexuelle, composante pourtant essentielle dans le développement et l'épanouissement de soi d'une grande majorité de personnes toutes orientations sexuelles confondues. L'hétérosexisme dépasse les gestes commis par d'autres personnes et exerce une contrainte sur les actions mêmes des personnes lesbiennes, gaies et bisexuelles. (2011, p. 129)

Dans ce contexte, ce sont les individus et les groupes qui sont en situation d'oppression qui sont les mieux placés pour comprendre les systèmes d'oppression et leurs différents mécanismes, puisqu'ils en subissent continuellement les impacts. Loin de s'inscrire automatiquement dans l'expérience des individus, une telle compréhension exige un processus de collectivisation et de conscientisation face aux structures politiques, sociales et économiques qui influencent leurs conditions de vie (Thompson, 2000). 


\section{Oppressions multiples, oppressions intercroisées et intersectionnalité}

"Comme les individus ont plusieurs identités, il est possible qu'ils soient en situation d'oppression en fonction de leur appartenance à plus d'un groupe social."
Comme les individus ont plusieurs identités, il est possible qu'ils soient en situation d'oppression en fonction de leur appartenance à plus d'un groupe social. Dès les années 1980, des féministes noires ont critiqué un certain discours féministe "blanc ", soulignant la nécessité de reconnaître leur réalité particulière et leur situation de double oppression comme femmes noires (hooks, 1984; Collins, 1990). À cet égard, hooks (1984) soutient que les femmes noires se situent au bas de l'échelle sociale; considérant le sexe, l'ethnicité et la classe sociale, elles auraient un statut inférieur à tous les autres groupes de la société. Dans le même sens, des auteures se sont intéressées à la double et à la triple infériorisation des femmes francophones vivant en contexte minoritaire (Cardinal, 1992) ou encore aux difficultés particulières auxquelles sont confrontés dans ce même contexte les nouveaux arrivants francophones (Kérisit, 1998). Kérisit relève certains des défis de l'intervention interculturelle en milieu minoritaire de langue française en Ontario :

Les barrières à la mobilité sociale sont plus hautes pour bien des nouveaux arrivants, en particulier la majorité venue des pays du Sud. Les diverses formes de discrimination raciale, s'exerçant à l'égard des personnes de race noire en particulier, l'exigence de l'anglais pour accéder à l'emploi et les conditions (légales) d'arrivée au Canada font que la mobilité au-delà de la frontière de la langue relève davantage de la stratégie de survie que d'une opposition de principe aux revendications des francophones nés au Canada. (1998, p. 95)

Dans cette perspective, Collins (1990) met de l'avant les concepts de " matrice des oppressions » et de "systèmes d'oppressions intercroisées " pour désigner les liens entre le sexisme, le racisme et d'autres systèmes d'oppression. Plus récemment, des auteures se sont inspirées des travaux des féministes noires et ont 
eu recours au concept d'intersectionnalité, initialement proposé par Crenshaw (1991), pour rendre compte de l'interaction entre les multiples oppressions vécues par les individus et les groupes. Selon Corbeil et Marchand (2006, p. 46),

le concept d'intersectionnalité apparait comme un outil d'analyse pertinent, d'une part, pour comprendre et répondre aux multiples façons dont les rapports de sexe entrent en interrelation avec d'autres aspects de l'identité sociale et, d'autre part, pour voir comment ces intersections mettent en place des expériences particulières d'oppression et de privilège.

\section{Domination et privilèges}

Bien que le concept d'oppression soit à la base des approches structurelles, il faut éviter de centrer toute notre attention sur les individus et les groupes qui sont en situation d'oppression,

"...cette tendance

à mettre l'accent sur

l'oppression plutôt

que sur la domination

risque de nous amener

à blâmer les individus

et les groupes qui

sont en situation

d'oppression pour les

difficultés auxquelles

ils sont confrontés et à

leur attribuer l'entière responsabilité pour les changements à apporter à leur situation. » occultant ainsi la domination et les privilèges qui y sont associés. Pease (2011) soutient que cette tendance à mettre l'accent sur l'oppression plutôt que sur la domination risque de nous amener à blâmer les individus et les groupes qui sont en situation d'oppression pour les difficultés auxquelles ils sont confrontés et à leur attribuer l'entière responsabilité pour les changements à apporter à leur situation.

En effet, si les individus sont toujours opprimés en fonction de leur appartenance à certains groupes sociaux, cette oppression existe aussi dans la mesure où d'autres groupes occupent une position de domination à laquelle sont associés un certain nombre de privilèges. Ainsi, le sexisme, le patriarcat, le colonialisme, le racisme, l'hétérosexisme, le capitalisme, le capacitisme et l'âgisme doivent donc être analysés sous l'angle de la domination, de manière à rendre visibles les individus et les groupes (les hommes, les "blancs", les personnes hétérosexuelles, les riches, etc.) qui retirent des privilèges de ces systèmes et qui ont avantage à ce qu'ils soient maintenus (Gil, 1998; Pease, 2011). Pease (2011) 
note que même s'ils sont généralement conférés aux individus en fonction de leur appartenance à certains groupes sociaux, ces privilèges sont souvent perçus positivement, comme étant le résultat d'efforts soutenus ou de la chance.

Ce sont donc les systèmes de domination qui doivent être ciblés pour arriver à un changement social, mais une telle démarche est souvent complexe. Si les individus et les groupes qui sont en situation d'oppression ne développent pas automatiquement une compréhension des systèmes d'oppression, les individus et les groupes qui en retirent des privilèges ont tout avantage à ce que ces mêmes systèmes demeurent cachés (Thompson, 2002; Pease, 2011).

\section{Applications en fonction de trois cibles d'intervention}

"Cette articulation de

l'intervention structurelle en fonction de trois cibles permet de développer "un processus continu qui passe de l'individu à l'action collective »."
Moreau (1987) identifie cinq pratiques qui sont au cœur de l'approche structurelle, soit la matérialisation des problèmes; leur collectivisation; la défense du client; le questionnement de l'idéologie dominante; et l'augmentation du pouvoir des clients. Si nous reconnaissons l'importance de ces dernières, nous proposons de notre côté, pour l'application des approches structurelles, des pistes qui portent sur trois cibles d'intervention : individuelle, groupe et collective.

Cette articulation de l'intervention structurelle en fonction de trois cibles permet de développer « un processus continu qui passe de l'individu à l'action collective " (Mayer et Panet-Raymond, 1991, p. 106). La cible individuelle a comme principal objectif de soulager les tensions et de répondre aux besoins immédiats identifiés par la personne, le groupe ou la collectivité. La cible groupe, quant à elle, vise la collectivisation des problèmes sociaux et la conscientisation aux facteurs structurels - les situations auxquelles les individus sont confrontés sont définies en termes collectifs, en lien avec les structures politiques, sociales et économiques. Enfin, la cible collective, en plus de promouvoir 
la réduction des inégalités sociales à travers la reconnaissance des droits individuels et collectifs, vise le changement social à moyen et à long terme, tout en reconnaissant que le conflit est au cœur de toute transformation importante (Moreau, 1987; Lundy, 2011).

Présenter à l'intérieur d'un seul article l'ensemble des éléments nécessaires pour mettre en pratique les approches structurelles relève de l'impossible. Nous ne proposons donc que des pistes visant leur application. Notons également que la distinction que nous établissons entre l'analyse et la pratique est artificielle, puisque l'analyse fait partie intégrante du processus d'intervention, car elle définit sur quoi l'action doit porter (Moreau, 1987; Lévesque et Panet-Raymond, 1994; Mullaly, 2007; 2011).

\section{Cible individuelle}

C'est la signification que nous accordons aux problèmes de la personne (le fait que nous les considérions dans le contexte social de la personne) et les buts que nous poursuivons par nos interventions (le soutien de la personne et l'obtention des ressources dont celle-ci a besoin pour agir sur son environnement) qui définissent la spécificité de la pratique individuelle en travail social par rapport à celle des autres professions. (Bourgon et Gusew, 2007, p. 123)

Dans une perspective structurelle, les intervenantes tentent d'abord d'établir des relations égalitaires avec les individus rencontrés. Pour ce faire, elles évitent de se positionner comme des " expertes ", résistant ainsi à la tendance à la professionnalisation et à la spécialisation de l'intervention sociale. Elles reconnaissent, au contraire, que les individus en situation d'oppression sont les mieux placés pour comprendre les systèmes d'oppression et leurs mécanismes, valorisent leur expérience et leur point de vue, soutiennent leur capacité à prendre des décisions et à faire des choix. De plus, les intervenantes reconnaissent que, même si les individus sont toujours opprimés en fonction de leur appartenance à certains groupes sociaux, chaque situation a un caractère unique 
"...les interventions

individuelles visent essentiellement à soulager les tensions et à répondre aux besoins immédiats, tels qu'identifiés par les individus eux-mêmes. »

"...les mesures mises en place pour répondre aux besoins immédiats des individus ne doivent pas être perçues comme des privilèges accordés aux individus, mais bien comme des actions visant le respect de leurs droits. ") et suscite des émotions et des réactions particulières chez les individus concernés (Lévesque et Panet-Raymond 1991; Carniol, 1992).

Même lorsqu'elles interviennent au niveau de la cible individuelle, les intervenantes privilégiant les approches structurelles évitent l'individualisation et la psychologisation des problèmes. Ainsi, les interventions individuelles visent essentiellement à soulager les tensions et à répondre aux besoins immédiats, tels qu'identifiés par les individus eux-mêmes (Moreau, 1987; Lundy, 2011). À ce propos, notons que la réponse aux besoins immédiats des individus (sécurité, nourriture, logement, etc.) constitue souvent une question de respect des droits fondamentaux, reconnus dans les politiques nationales et internationales. Par exemple, le Canada a signé en 1976 le Pacte international relatif aux droits économiques, sociaux et culturels (PIDESC) qui reconnait les droits suivants pour toutes et tous : le droit à un niveau de vie suffisant (incluant le logement, les vêtements et la nourriture), le droit de jouir du meilleur état de santé possible, le droit à des conditions de travail justes et à l'éducation primaire gratuite (Greason, 2008). Ainsi, les mesures mises en place pour répondre aux besoins immédiats des individus ne doivent pas être perçues comme des privilèges accordés aux individus, mais bien comme des actions visant le respect de leurs droits.

Il s'agit d'objectifs à court terme, qui ne doivent pas être confondus avec les objectifs à plus long terme (Gil, 1998). Cependant, lorsque l'urgence de la situation est résorbée, les individus sont invités à prendre conscience du fait qu'ils ne sont pas les seuls à être confrontés à de telles difficultés, que certaines structures, politiques, sociales ou économiques, peuvent être à la base de ces difficultés et contribuer au maintien de la situation problématique. Les intervenantes peuvent partager leur propre compréhension des problèmes avec les individus et elles peuvent s'adonner avec eux à un examen critique de certains discours politiques, ainsi que de certaines politiques et pratiques sociales et économiques. Cet examen peut mettre l'accent sur la scène locale, nationale ou internationale, ou encore sur les politiques et les pratiques en place au sein de l'organisation. 


\section{Cible de groupe}

"...l'intervention de

groupe permet une

réelle collectivisation

des problèmes et une

conscientisation en

ce qui a trait aux

facteurs politiques, sociaux et économiques

qui ont contribué au développement ou au maintien des ces problèmes. "
Enfin, les intervenantes peuvent inviter les individus à se joindre à un groupe dont les participantes ont un parcours similaire ou partagent un intérêt commun, dans le but de poursuivre une démarche de conscientisation et de collectivisation aboutissant ultimement à une action collective visant le changement social.

[La conscientisation] est un processus dans lequel des individus, en tant que sujets connaissants, et non en tant que bénéficiaires, approfondissent la conscience qu'ils ont à la fois de la réalité socioculturelle qui modèle leur vie et de leur capacité de transformer cette réalité. (Freire, 1970,p.453)

Dans une perspective structurelle où les intervenantes souhaitent éviter l'individualisation et la psychologisation des problèmes, l'intervention de groupe permet une réelle collectivisation des problèmes et une conscientisation en ce qui a trait aux facteurs politiques, sociaux et économiques qui ont contribué au développement ou au maintien des ces problèmes. Les intervenantes mettent donc en place des groupes dont les participantes ont un parcours similaire ou partagent un intérêt commun, et elles accompagnent ces groupes dans une analyse structurelle des problèmes auxquels ces dernières sont confrontées. Comme le souligne Ninacs (2008, p. 79),

l'avantage du groupe par rapport à l'intervention individuelle tient à ce que l'activité collective permet d'élever plus facilement le niveau de conscience critique et au fait que le groupe permet une relation de collaboration entre les membres, fondée sur l'engagement mutuel et la réciprocité, ainsi que sur le partage du pouvoir et de la lutte à mener.

Tout comme pour l'intervention individuelle, les intervenantes privilégiant une perspective structurelle évitent de se positionner 
"Un tel processus de collectivisation et de conscientisation est en lui-même un processus politique favorisant une plus grande justice sociale."

\section{Cible collective}

"Les actions qui relèvent de la cible collective visent un changement social à moyen et à long terme. " comme des "expertes" face aux participantes. À cet égard, Mullender et Ward (1991) proposent un modèle de groupe autogéré qui illustre bien ce à quoi devrait correspondre l'intervention de groupe dans une perspective structurelle. Selon ce modèle, les membres d'un groupe sont les principaux acteurs de la démarche, de la phase de planification à celle de travail, en passant par l'appréciation continue de la démarche. Ainsi, ce modèle propose une réelle appropriation du pouvoir par les participantes, et ce, tout au long du processus d'intervention.

Un tel processus de collectivisation et de conscientisation est en lui-même un processus politique favorisant une plus grande justice sociale. Les participantes peuvent elles-mêmes exprimer leur surprise lorsqu'elles réalisent que d'autres personnes sont confrontées à des difficultés similaires. De plus, une mise en commun d'expériences individuelles peut éveiller une conscience collective et mener éventuellement à certaines actions ou revendications en lien avec les structures politiques, sociales et économiques (Shragge, 2006).

L'organisation communautaire qui vise à réaliser le changement social commence avec cette impulsion [l'indignation]. Apprendre quoi faire de notre colère et notre révolte, les investir dans les luttes locales, leur donner une plus grande portée et les entretenir pour alimenter notre élan en faveur de la justice sociale : tel est le défi à relever. (Shragge, 2006, p. 233)

Les actions qui relèvent de la cible collective visent un changement social à moyen et à long terme. À l'instar de Shragge (2006), nous croyons qu'un changement social d'envergure nécessite d'abord l'indignation, puis l'engagement et le militantisme. Il s'agit donc pour les intervenantes de mobiliser les individus et les groupes dans l'élaboration et la mise en œuvre d'actions et de revendications. Dans ce contexte, les intervenantes 
sont des mobilisatrices, des accompagnatrices, des médiatrices, des militantes et des activistes. Tout en s'associant à la démarche, elles doivent respecter le rythme propre à chaque groupe et demeurer vigilantes pour ne pas s'approprier le projet comme s'il s'agissait de leur propre initiative plutôt que d'une démarche collective (Shragge, 2006).

Tout comme pour la cible individuelle, les actions et revendications collectives peuvent mettre de l'avant les droits collectifs. Comeau (2012) souligne en effet l'importance de la promotion et de la défense de ces droits. La promotion des droits fait référence à «l'articulation d'une série d'actions visant à faire inscrire dans des règles, des lois et dans des coutumes des principes respectant l'intégrité de certaines personnes » tandis que la défense des droits fait plutôt référence à " la programmation d'activités visant à faire respecter les règles et les lois s'appliquant dans un domaine " (Comeau, 2012, p. 78).

Par ailleurs, les actions entreprises au niveau de la cible collective peuvent permettre d'éveiller la conscience sociale et politique chez des individus et des groupes qui ne sont pas directement affectés par la situation dénoncée, favorisant ainsi une plus grande solidarité au sein de la société.

\section{Habiletés et attitudes nécessaires en intervention structurelle}

Les intervenantes qui privilégient une perspective structurelle utilisent diverses techniques d'intervention qui sont à la base de tout processus de relation d'aide, incluant l'écoute active, la communication non verbale et le reflet (Lévesque et Panet-

"Il y a néanmoins des

habiletés et des attitudes

qui sont davantage

associées aux approches

structurelles, dont

plusieurs s'inscrivent

dans la tradition

des pratiques de

conscientisation."
Raymond, 1987; Moreau, 1987). Il y a néanmoins des habiletés et des attitudes qui sont davantage associées aux approches structurelles, dont plusieurs s'inscrivent dans la tradition des pratiques de conscientisation (Ampleman, et collab., 1994).

Tout d'abord, les intervenantes doivent s'engager dans une réflexion concernant leur position sociale et les zones d'oppression et de privilèges qui y sont associées, ainsi que sur les enjeux de pouvoir dans leurs relations avec les individus, les groupes et les collectivités. Cette réflexion est nécessaire pour qu'elles soient en 
"Les intervenantes démontrent aussi une capacité d'indignation face à toutes les manifestations de l'oppression; c'est cette capacité qui leur permet de se mobiliser et d'entreprendre une démarche de changement." mesure de développer des relations égalitaires avec les personnes rencontrées.

Les intervenantes démontrent aussi une capacité d'indignation face à toutes les manifestations de l'oppression; c'est cette capacité qui leur permet de se mobiliser et d'entreprendre une démarche de changement. Elles doivent aussi croire que le changement social est possible. Cet engagement dépasse d'ailleurs le cadre de leur travail, puisqu'il s'agit d'une implication à la fois professionnelle et personnelle.

Compte tenu de ce qui a été présenté ci-dessus, les intervenantes qui privilégient une perspective structurelle doivent démontrer une bonne capacité d'analyse critique, incluant la dimension historique et les dimensions politiques, sociales et économiques des problèmes auxquels les individus, les groupes et les collectivités sont confrontés. Cela permet d'éviter l'individualisation et la psychologisation des problèmes sociaux. Elles doivent aussi avoir une bonne connaissance des droits individuels et collectifs - en matière de promotion et de défense de droits, le Pacte international relatif aux droits économiques, sociaux et culturels (PIDESC) est une bonne assise sur laquelle appuyer les revendications - et elles doivent être en mesure de promouvoir et de défendre ces droits, tant à l'extérieur qu'à l'intérieur de leur organisation.

Les intervenantes qui privilégient une perspective structurelle montrent également des habiletés tant sur le plan de l'intervention individuelle que sur celui de l'intervention de groupe et de l'action collective. Cette polyvalence exige différentes connaissances propres à chacune de ces trois méthodes d'intervention. Elles doivent aussi faire preuve de flexibilité et de créativité, et doivent constamment remettre en question leur façon de voir, de faire et d'être, afin de respecter la culture propre à chaque individu, groupe ou collectivité.

Finalement, les intervenantes doivent bien connaître les ressources du milieu, pour être en mesure de référer les personnes et les groupes accompagnés, mais aussi pour faciliter l'établissement de collaborations et de coalitions. 


\section{Conclusion}

"...le travail social est historiquement parlant la seule profession de relation d'aide qui a réellement placé la justice sociale au coenr de ses valeurs et de ses préoccupations. Ainsi, il n'y a aucun doute concernant la pertinence des approches structurelles dans le champ de l'intervention sociale."
Vingt-cinq ans après la publication de l'article de Moreau (1987), si le paysage de l'intervention et le contexte politique, social et économique dans lequel les interventions s'inscrivent ont changé de manière significative, l'application des approches structurelles requiert toujours une certaine énergie et comporte toujours un certain risque. En effet, plusieurs tendances récentes dans le champ de l'intervention sociale vont à l'encontre d'une perspective structurelle. Ces tendances, telles l'individualisation et la psychologisation des problèmes, ne se limitent pas au champ de l'intervention sociale, elles se manifestent également dans les discours politiques, dans les médias, et partout ailleurs.

Néanmoins, Lundy (2011) souligne que le travail social est historiquement parlant la seule profession de relation d'aide qui a réellement placé la justice sociale au cœur de ses valeurs et de ses préoccupations. Ainsi, il n'y a aucun doute concernant la pertinence des approches structurelles dans le champ de l'intervention sociale. Plus encore, nous estimons qu'il est nécessaire pour les intervenantes sociales de reconnaitre que les situations individuelles sont intimement reliées aux structures politiques, sociales et économiques et que la réalisation de changements significatifs dans les situations auxquelles les individus sont confrontés exige des changements dans ces mêmes structures. Nous croyons que les interventions devraient toujours s'inscrire dans une visée de changement social.

Cela soulève évidemment la question suivante : est-ce que les intervenantes, lorsqu'elles accompagnent les individus et les groupes et les aident à composer avec diverses situations difficiles, sont aussi des agentes de changement social? (Gil, 1998) En réponse, nous croyons que notre articulation de l'intervention structurelle en fonction de trois cibles permet de situer les interventions individuelles dans un processus continu qui à moyen ou à long terme passe à l'action collective.

Nous osons croire que des travailleuses sociales sont toujours prêtes à relever ce défi : agir pour une société juste et équitable 
pour tous les individus, les groupes et les collectivités. Ces efforts devraient être soutenus par les associations professionnelles et par les institutions de formation et de recherche en service social, qui peuvent aussi mettre en évidence la pertinence, voire la nécessité, d'une analyse structurelle des problèmes sociaux.

\section{Note}

1 Par souci de cohérence avec l'analyse proposée dans cet article, les auteures ont privilégié le féminin pour désigner à la fois les intervenantes et les intervenants.

\section{Bibliographie}

AMPLEMAN, Gisèle, et collab. (1994). La conscientisation : définition et principes d'action, Les cahiers de la conscientisation, Numéro 1, Le Collectif québécois d'édition populaire, Québec, $21 \mathrm{p}$.

AUCLAIR, René (2000). «Valeurs et éthique en travail social », dans Jean-Pierre Deslauriers et Yves Hurtubise, Introduction au travail social, Québec, Presses de l'Université Laval, 426 p.

BASTIEN CHARLEBOIS, Janik (printemps 2011). "Au-delà de la phobie de l'homo : quand le concept d'homophobie porte ombrage à la lutte contre l'hétérosexisme et l'hétéronormativité ", Reflets : revue d'intervention sociale et communautaire, Vol. 17, $\mathrm{N}^{\circ}$ 1, p. 122-149.

BOURGON, Michèle, et Annie GUSEW (2007). "L'intervention individuelle en travail social » dans Jean-Pierre Deslauriers et Yves Hurtubise (2007), Introduction au service social, Québec, Les Presses de l'Université Laval, coll. "Travail social », 382 p.

BRETON, Judith (1993). "Supervision pédagogique dans l'approche de conscientisation, une expérience de formation en techniques de travail social ", Service social,Vol. 42, No 2, p. 143-162.

CARDINAL, Linda (1992). «La recherché sur les femmes francophones vivant en milieu minoritaire : un questionnement sur le féminisme ", Recherches féministes, Vol. 5, p. 5-29.

CARNIOL, Ben (1992). "Structural social work: Maurice Moreau's challenge to social work practice ", Journal of progressive human services, Vol. 3, No 1, p. 1-20.

CODERRE, Cécile, et Joanne HART (2003). « Pratiques d'intervention féministe auprès des femmes survivantes d'agression à caractère sexuel : le contexte franco-ontarien ", Reflets : revue ontaroise d'intervention sociale et communautaire, Vol. 9, № 1, p. 186-210.

COLLINS, Patricia H. (1990). Black feminist thought: Knowledge, consciousness, and the politics of empowerment, Routledge, New York, 335 p.

COMEAU,Yvan (2012). «Les stratégies d'intervention sociopolitique favorables à l'action collective ", Reflets : revue d'intervention sociale et communautaire, Vol. 18, No 1, p. 61-90.

CORBEIL, Christine, et Isabelle MARCHAND (2006). «Penser l'intervention féministe à l'aune de l'approche intersectionnelle : défis et enjeux ", Nouvelles pratiques sociales, Vol. 19, p. 40-57.

COUTURIER,Yves, et Sébastien CARRIER (2003). « Pratiques fondées sur les données probantes en travail social : un débat émergent ", Nouvelles pratiques sociales, Vol. 16, № 2, p. 68-79.

CRENSHAW, Kimberlé (1991). "Mapping the margins: Intersectionnality, identity politics and violence against women ", Stanford Law Review, No 43, p. 1241-1298. 
DOMINELLI, Lena (2002). Feminist social work theory and practice, Houndmills, Palgrave Macmillan, $208 \mathrm{p}$.

DUBOIS, Madeleine, et Marie-Luce GARCEAU (2000). "L'évolution du travail social : une histoire à suivre - Entrevue avec Roland Lecomte ", Reflets : revue ontaroise d'intervention sociale et communautaire, Vol. 6, No 1, p. 18-34.

FREEMAN, Bonnie (2007). "Indigenous pathways to anti-oppressive practice ", dans D. Baines (dir.), Doing anti-oppressive practice: Building transformative politicized social work, Halifax, Fernwood Publishing, p. 95-127.

FISH, Julie (2006). Heterosexism in health and social care, Houndmills, Palgrave Macmillan, 248 p.

FREIRE, Paulo (1970). Pedagogy of the oppressed, Continuum International Publishing Group, New York, $183 \mathrm{p}$.

FRYE, Marilyn (1983). The Politics of reality: Essays in feminist theory, The Crossing Press, $175 \mathrm{p}$.

GIL, David G. (1998). Confronting injustice and Oppression: Concepts and strategies for social workers, Columbia University Press, New York, 165 p.

GILL, Louis (2004). Le néolibéralisme, Montréal, Chaire d'études socio-économiques de l'UQAM, 84 p.

GRAMMOND, Sébastien (2006). "La mémoire du colonialisme et les droits des autochtones du Canada ", dans Serge Jaumain et Éric Remacle (dirs.), Mémoire de guerre et construction de la paix, Brussels, P.I.E.-Peter Lang, p. 117-135.

GREASON,Vincent (2008). "Un filet de protection troué pour faire face à la crise économique ", Bulletin spécial : Quels défis pour les droits humains de la Ligue des droits et libertés, Montréal, p. 7-9.

hooks, bell (1984). Feminist theory from margin to center, South End Press, $174 \mathrm{p}$.

HAUT-COMMISSARIAT DES NATIONS-UNIES AUX DROITS DE L'HOMME (1966). "Pacte international relatif aux droits économiques, sociaux et culturels ", réf. du 12 février 2013, http://www2.ohchr.org/french/law/cescr.htm

JOHNSON, Holly, et Myrna DAWSON (2011). Violence against women in Canada: Research and policy perspectives, Toronto, Oxford University Press, $240 \mathrm{p}$.

KÉRISIT, Michèle (1998). "Les défis de l'intervention interculturelle en milieu minoritaire de langue française en Ontario ", Reflets : Revue ontaroise d'intervention sociale et communautaire, Vol. 4, No 1, p. 75-99.

KRANE, Julia, et Rosemary CARLTON (2012). « Une pratique à la croisée des chemins : comprendre les femmes en tant que mères en maison d'hébergement ", dans Simon Lapierre et Dominique Damant (dirs.), Regards critiques sur la maternité dans divers contextes sociaux, Sainte-Foy, Presses de l’Université du Québec, p. 185-204.

LAMOUREUX, Henri (2010). La pratique de l'action communautaire autonome, Presses de l'Université du Québec, Québec, 116 p.

LAVOIE, Joscelyne, et Jean PANET-RAYMOND (2011). La pratique de l'action communautaire, Presses de l'Université du Québec, 416 p.

LÉVESQUE, Justin, et Jean PANET-RAYMOND (1994). «L'évolution et la pertinence de l'approche structurelle dans le contexte social actuel ", Service social,Vol. 43, No 3, p. 23-39.

LÉVESQUE, Justin, et Jean PANET-RAYMOND (1987). «L'apprentissage d'une approche intégrée : l'expérience de l'approche structurelle ", Service social,Vol. 36, № 2-3, p. 413-433. 
LUNDY, Colleen (2011). Social work, social justice and human rights, Toronto, University of Toronto Press, $384 \mathrm{p}$.

MAYER, Robert, et Jean PANET-RAYMOND (1991). "L'action communautaire de défense des droits sociaux » dans Laval Doucet et Louis Favreau (1991), Théorie et pratiques en organisation communautaire, Québec, Presses de l'Université du Québec, p. 97-118.

MOLGAT, Marc (2007), "Définir le travail social... », dans Jean-Pierre Deslauriers etYves Hurtubise (2007), Introduction au service social, Québec, Les Presses de l'Université Laval, coll. «Travail social », $382 \mathrm{p}$.

MOREAU, Maurice (1987). "L'approche structurelle en travail social : implications pratiques d'une approche intégrée conflictuelle », Service social,Vol. 36, № 2-3, p. 227-247.

MULLALY, Bob (2007). The new structural social work, Ontario, Oxford University Press, 398 p.

MULLALY, Bob (2010). Challenging oppression and confronting privilege, Ontario, Oxford University Press, $356 \mathrm{p}$.

MULLENDER, Audrey (2002). "Persistent oppressions:The example of domestic violence ", dans R. Adams, L. Dominelli et M. Payne (dirs.), Critical practice in social work, Houndmills, Parlgrave Macmillan, p. 63-71.

MULLENDER, Audrey (2003) "Conclusion: Where does this leave us? » dans Maria Cohen et Audrey Mullender, (dirs.), Gender and groupwork, London, Routledge, p. 192-208.

MULLENDER, Audrey, et Dave WARD (1991). Self-directed groupwork: Users take action for empowerment, London, Whiting \& Birch, 194 p.

MURRAY, Kate M., et Steven F. HICK (2010). «Structural social work :Theory and process », dans S. F. Hick, et collab. (dirs.), Structural social work in action: Examples in practice, Toronto, Ontario, Canadian Scholars' Press, p. 3-25.

NINACS, William A. (2008). Empowerment et intervention : développement de la capacité d'agir et de la solidarité, Québec, Les presses de l'Université Laval, 140 p.

PAYNE, Malcolm (2005). Modern social work theory, Houndmills, Palgrave Macmillan, 366 p.

PEASE, Bob (2009). «From evidence-based practice to critical knowledge in post-positivist social work ", dans June Allan, Linda Briskman et Bob Pease (dirs.), Critical social work, Theories and practices for a socially just world, Allen \& Unwin, p. 45-57.

PEASE, Bob (2010). Undoing privilege: Unearned advantage in a divided world, Zed Books Ltd, London, $224 \mathrm{p}$.

PRUD’HOMME, Diane (2011). « La violence conjugale : quand la victimisation prend des allures de dépendance affective! », Reflets : revue d'intervention sociale et communautaire,Vol. 17, № 1, p. 180-190.

RENÉ, Jean-François (2009). "L'individualisation de l'intervention dans les organismes communautaires : levier ou barrière à la prise en charge démocratique? ", Nouvelles pratiques sociales, Vol. 22, No 1, p. 111-124.

SHRAGGE, Eric (2006). Action communautaire : dérives et possibles, Les éditions écosociété, Montréal, $242 \mathrm{p}$. ST-AMAND, Nérée (2003). "Interventions opprimantes ou conscientisantes? » Reflets : Revue ontaroise d'intervention sociale et communautaire, Vol. 9, № 2, p. 139-162.

THIBAULT, Sylvie (2011). "Nature et fondements de l'intervention sociale personnelle " dans Daniel Turcotte et Jean-Pierre Deslauriers (2011), Méthodologie de l'intervention sociale personnelle, Québec, Les Presses de l’Université Laval, coll. «Travail social », p. 9-29.

THOMPSON, Denise (2001). Radical feminism today, London, Sage, 176 p. 
TURCOTTE, Daniel, et Jocelyn LINDSAY (2008). L'intervention sociale auprès des groupes, Boucherville, Gaëtan Morin, 208 p.

VAILLANCOURT, Yves (2001). «Le logement public : enjeux et perspectives ", Nouvelles pratiques sociales, Vol. 14, N¹, p.1-7.

YOUNG, Iris (1990). Justice and the politics of difference, Princeton University Press, $304 \mathrm{p}$. 